\title{
TOWARDS ASSISTED LIVING VIA PROBABILISTIC VITAL-SIGN MONITORING IN THE HOME
}

\author{
D.A. Clifton*, L. Clifton*, M. Alvi*, S. Khalid*, \\ D. Meredith ${ }^{\dagger}$, J. Price ${ }^{\ddagger}$, P. Watkinson ${ }^{\S}$, L. Tarassenko* \\ *Institute of Biomedical Engineering, Department of Engineering Science, University of Oxford, Oxford, UK \\ †Nuffield Department of Clinical Medicine, University of Oxford, Oxford, UK \\ $\$$ Nuffield Department of Anaesthetics, University of Oxford, Oxford, UK \\ $\S$ John Radcliffe Hospital, Oxford, UK
}

Corresponding author: david.clifton@eng.ox.ac.uk

Keywords: Telehealth, Home Monitoring, Data Fusion

\begin{abstract}
This paper describes the development of a reliable multisensor data fusion system for monitoring patient vital-signs in the home. Initial investigatory work has taken place using ambulatory hospital patients, in the Oxford Cancer Hospital. Our monitoring approach is based on a probabilistic model of normality learned from a data-set of vital signs acquired from a representative group of high-risk patients. Alerts are provided to carers whenever patient vital signs are deemed "abnormal" with respect to the model of normality. We show examples of how this approach correctly detects physiological deterioration in the target patient group, and describe future work in further validation of the technology in home monitoring applications.
\end{abstract}

\section{Introduction}

With major UK hospitals becoming ever-more focussed on the care of high-acuity patients, those patients of a lower acuity must be treated in a community hospital or other local community setting. In collaboration with the Oxford Radcliffe Hospitals Trust, we are initiating a novel countywide scheme in which multidisciplinary Emergency Medical Units (EMUs) are created in key community hospitals, which will use data fusion systems for the monitoring of these lower-acuity patients, who would otherwise have been referred to a major NHS hospital. Patients deemed fit for discharge to their own home will be monitored remotely using data fusion systems via telemetry, with clinicians able to monitor cohorts of patients in conjunction with a dedicated team of home nursing staff.

Wireless sensor technology for monitoring patient vital signs in the home is now available. However, the real challenge for this technology is not measuring vital signs, but determining how they should be processed, such that the number of false alarms generated is sufficiently low for the system to be used to monitor home patients in practice. We argue that home monitoring of vital signs will only be possible with a combination of multiple wireless sensors and data fusion techniques, to drive the rate of false alerts down to an acceptably low level and deliver the potential benefits of remote monitoring, both in terms of patient outcomes and health economics.

\subsection{Overview of this paper}

We have previously shown that the integration of continuously-monitored parameters in data fusion models can provide early warning of adverse events. This has been demonstrated both in jet engine health monitoring [3] and in the monitoring of acutely ill hospital patients [8]. Our proposed patient monitoring approach is based on a probabilistic model of normality learned from a dataset of vital signs acquired from a representative group of high-risk hospital patients. This system alerts the nursing staff whenever the combination of vital sign parameters is indicative of physiological abnormality, as described in Section 2.

Such an approach is readily applicable to monitoring patients in the home, with the use of appropriate wireless sensors. This paper describes an investigation of a data-fusion approach to patient monitoring using data acquired from ambulatory patients; this approach contrasts with previous studies, which monitored critical patients who are confined to a hospital bed, and who are tethered to a fixed, conventional bed-side patient monitor. Section 3 describes an on-going investigative study in which ambulatory patients within a post-surgical ward of the Oxford Cancer Hospital are monitored using the proposed data fusion approach, with results presented in Section 4.

This patient group was selected in order to be able to investigate the challenges introduced by moving from bedpatients to ambulatory patients, where the location of the hospital ward provides sufficient numbers such that a suitable 
large population can be studied, prior to its subsequent implementation in home-based studies, which are described in Section 5.

\section{Probabilistic data fusion}

A multimodal, multivariate model of normality may be constructed from a large dataset of "normal" vital-sign data acquired from patients deemed to be representative of the patient cohort to be studied. A training set $\mathbf{X} \in \mathbb{R}^{D}$, with dimensionality $D=5$ (corresponding to heart rate, breathing rate, blood-oxygen saturation, temperature, and blood pressure), was obtained from monitoring a large number of high-risk patients for over 3,000 hours. Each vital sign was standardised with respect to the mean and variance of that vital sign in the training set. Parzen's window method [2] was used to estimate the distribution of the training set in $\mathbb{R}^{5}$,

$$
p(\mathbf{x})=\frac{1}{N(2 \pi)^{5 / 2} \sigma^{5}} \sum_{i=1}^{N} \exp \left(-\frac{\left|\mathbf{x}-\boldsymbol{\mu}_{i}\right|^{2}}{2 \sigma^{2}}\right)
$$

which is a kernel-based approach, and which averages the responses of a multivariate Gaussian kernel placed on each of $i=1 \ldots N$ locations $\boldsymbol{\mu}_{i}$. It is conventional to set $\left\{\boldsymbol{\mu}_{i}\right\}=\mathbf{X}$, such that a kernel is placed on each data-point in the training set; however, the very number of training data in $\mathbf{X}$ requires that they are first summarised to prevent the processing of $|\mathbf{X}|$ Gaussian activations for each test data-point, $\mathbf{x}$. The $K$-means algorithm was used to reduce the size of the dataset to $K=500$ cluster centres, and the $N=400$ centres closest to the centroid of $\mathbf{X}$ were subsequently used to locate the Gaussian kernels, $\boldsymbol{\mu}_{i}$. This assumed that these innermost cluster centres represented "normality" in the patient data, based on prior knowledge that over $80 \%$ of the training patients suffered no physiological deterioration [4]. The density estimator in Equation (1) assumes that each Gaussian kernel is isotropic and identical in covariance, such that all may be described by a single variance parameter (sometimes termed the bandwidth), $\sigma$. This parameter was estimated using the nearest-neighbour method proposed in [1].

\subsection{Novelty scores}

Noting that the probability density takes low values in regions of $\mathbb{R}^{5}$ which are far from the locations of the "normal" training data in $\mathbf{X}$, we define a novelty score,

$$
z(\mathbf{x})=\ln \frac{1}{p(\mathbf{x})}=-\ln p(\mathbf{x})
$$

such that test data $\mathbf{x}$ take larger values $z(\mathbf{x})$ as their support on $p$ decreases, representing more "abnormal" vital signs

\subsection{Testing new data}

As new vital signs are acquired, a vector $\mathbf{x}$ may be compared with the model of normality using Equation (1), and a novelty score $z(\mathbf{x})$ subsequently calculated using Equation (2), which may occur as frequently as $1 \mathrm{~Hz}$ in most conventional (bedside) monitoring systems.
A threshold $\kappa$ is placed on the novelty score such that "normal" and "abnormal" vital signs take values $z(\mathrm{x}) \leq \kappa$ and $z(\mathrm{x})>\kappa$, respectively. Noting that vital-sign data may be acquired at a high rate, and are sensitive to movement and sensor artefact that may introduce noise onto $\mathbf{x}$, and thus onto $z(\mathbf{x})$, some low-pass filtering is required to remove sharp transients in vital-sign data that are too rapid to correspond to physiological behaviour. In this paper, we show novelty scores $\mathrm{z}(\mathrm{x})$ after median filtering with a window length of $W$, where the value of $W$ is selected to correspond to a duration of approximately 30s, during which period it is assumed that any variations in vital signs are due to noise and artefact, and not physiological causes.

\section{A study for monitoring ambulatory patients}

Ambulatory patients in the Oxford Cancer Hospital were selected for the basis of an investigative study, where the cohort was recruited from subjects who had recently undergone surgery to remove upper-gastrointestinal cancer and who were recovering in a "step-down" ward. These patients remain in bed for the first day after surgery, and are then encouraged to walk around the ward for the remainder of their stay in hospital, which is expected to be approximately 4 to 5 days.

\subsection{Monitoring infrastructure}

The infrastructure for monitoring patients in the study is shown in Figure 1.

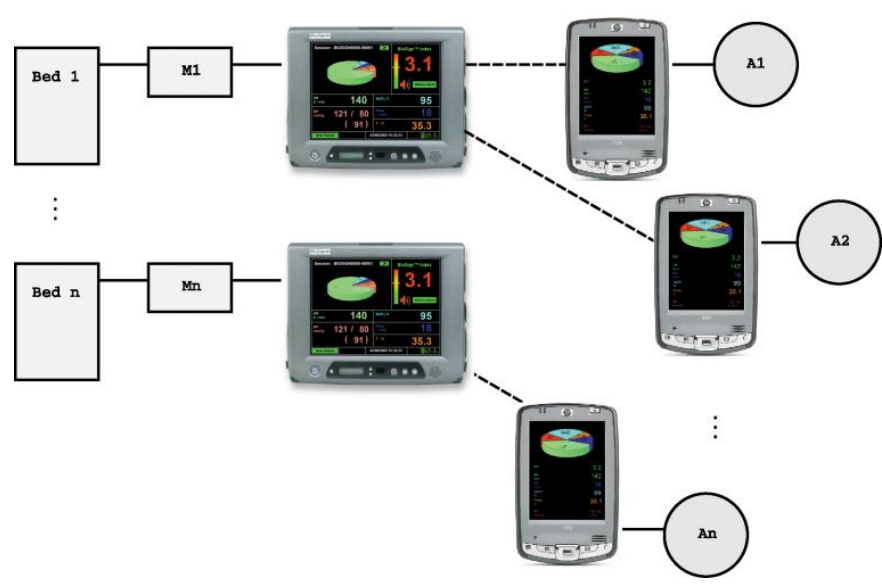

Figure 1: Monitoring infrastructure for the investigative study, in which patients connected to bed-side monitors $(M n)$ and those connected to ambulatory monitors $(A n)$ are monitored in parallel using a distributed wireless architecture.

The figure shows that bed-side monitors are used to collect data from patients during their first day of recovery, which may then be used to compare to the quality of data acquired from the ambulatory monitors that are subsequently used after the first day. 


\subsection{Vital-sign acquisition}

Ambulatory monitoring consists of a portable pulse oximeter, worn on the patient's finger or ear-lobe, which reports the heart rate (HR) and the saturation of oxygen in the blood $\left(\mathrm{SpO}_{2}\right)$. The breathing rate may be estimated from the amplitude and frequency modulation of the heart rate caused by respiration. These modulations are due to independent physiological phenomena, believed to be due to mechanical physiological pressures and respiratory sinus arrhythmia, for amplitude and frequency modulation, respectively. Thus, a "fused" estimate of breathing rate may be obtained, based on the two modulating signals, as described in [5]. Data from the pulse oximeter are transmitted to a belt-worn PDA via Bluetooth, which then transmits data to a Central Station via a wireless network.

We observe in passing that patients are highly resistant to wearing ECG electrodes for continued periods, and the use of a single device (the pulse oximeter) from which three vital signs may be acquired was deemed sufficient. We anticipate similar resistance to wearing ECG electrodes in home patients, and note that the pulse oximeter may be ideal in that it is easy to apply by the patient, whereas ECG electrodes require some familiarity to locate correctly.

Patient temperature and blood pressures were measured intermittently by ward staff. We note that skin-mounted temperature sensors are typically unreliable, due to changes in contact between the skin and the sensor that result in significant (and random) offsets between the measured temperature and actual patient temperature. Furthermore, the variance of temperature data acquired from skin-mounted probes is usually so significant that the signal is too noisy to be used reliably. In the home monitoring scenario, it is anticipated that temperature and blood pressure can be monitored with a similar accuracy to that achieved by ward staff by asking the patients to measure their own blood pressure and temperature once or twice per day using easy-touse models designed for home use.

\section{Results}

\subsection{Existing care}

The existing standard of ward- and home-based care is to take periodic observations of patient vital signs, often manually. These vital signs may then be scored using an "early warning score" (EWS), in which each vital sign is scored according to a set of pre-defined criteria, and then the scores are summed [7]. If any one of the scores for the individual vital signs, or the sum of all scores, exceeds pre-defined thresholds, then a "trigger" is deemed to have occurred, and the patient is reviewed. This policy of manual, low-frequency scoring has been mandated for use throughout hospitals in the NHS by the National Institute for Clinical Excellence [6]. In all published EWS systems to date, the scores and thresholds are not based on evidence, and are typically determined using expert opinion, which varies from hospital to hospital (and even from ward to ward). Thus, results vary significantly between institutions.

Furthermore, due to their manual nature, such EWS systems are prone to human error in (i) measuring vital signs, (ii) correctly assigning scores to vital signs, (iii) summing the scores to give a total score, and (iv) identifying that scores have exceeded pre-defined thresholds.

As a comparator to the electronic monitoring in this investigative study, vital sign observations (and the scores assigned to each) made by ward staff were recorded, and concerted from their paper-based form into an electronic format for analysis using double data-entry by independent teams. 812 manual observations were obtained from 166 patients. Retrospective analysis showed that between $2.3 \%$ and $12.8 \%$ of vital signs were not measured, and, of those vital signs measured, approx. $11.5 \%$ were subsequently not scored, while $15.1 \%$ of the scores were incorrect. $2.7 \%$ of the erroneous scores were "critical", where a score that should have been in excess of an alert threshold (and thus result in clinical review of the patient) were incorrectly scored such that they fell below the threshold (which would not result in clinical review).

Figure 2 shows a visualisation of 5-dimensional, manuallyobserved vital signs, projected into 2 dimensions using principal components analysis [2], using the two principal components of the data set.

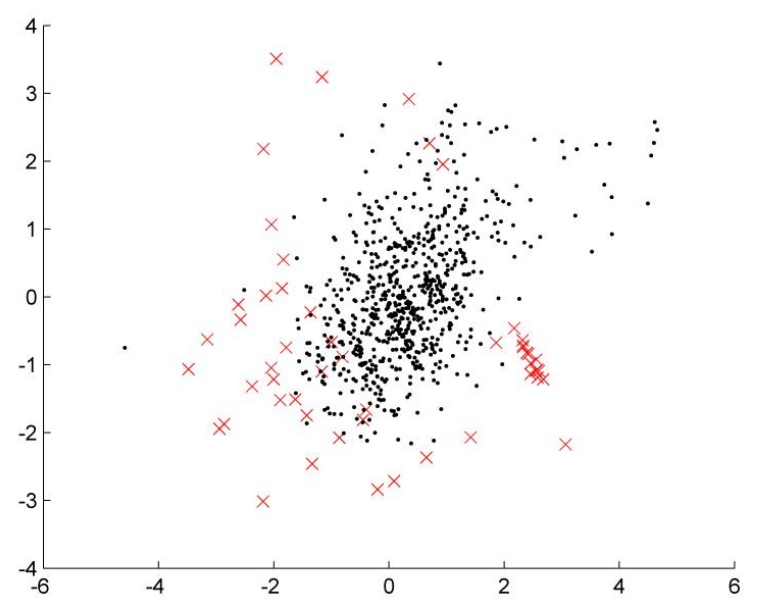

Figure 2: PCA visualisation of all manual vital-sign observations, with those that should have been scored above alerting thresholds, but which were incorrectly scored, shown in red.

The figure shows that the missed alerts fall into the more extreme regions of the projected space, corresponding to "abnormal" physiology, away from the large cluster of "normal" data.

Thus, our results suggest that this existing standard of care could be improved upon by the automated monitoring system, in which alerts are automatically generated. 


\subsection{Automated monitoring}

The majority of patients in the investigative study recovered normally, and were discharged home. However, a small proportion experienced physiological deterioration such that they were admitted to the intensive care unit (ICU).

Figure 3 shows a case study of a deteriorating patient, who was connected to monitoring equipment for two days prior to an emergency ICU admission that took place on 29 April (and which is shown as the large grey area in the figure). It may be seen that the ambulatory monitor provides vital-sign measurements that agree with manual observations of vital signs made by ward staff. However, the estimation of the breathing rate shows considerable variance with respect to the manual observations. This is due to the fact that manual observations of breathing rate are accurate, with the ward clinician typically measuring the number of chest-wall movements in order to calculate the breathing rate. In comparison, the estimation method employed by the automated system, as described in Section 3, is less robust. However, the estimate is sufficient such that an indication of patient condition can be provided, and the general trend of the breathing rate can be inferred by filtering the estimated signal.

The figure also shows novelty scores $z(\mathbf{x})$ determined using Equation (2), and those novelty scores obtained after application of a median filter, as described in Section 2. It may be seen that the variance of the breathing rate estimate is superimposed upon the novelty score, but that the trend can be recovered by the median filter. Peaks in the smoothed novelty threshold exceed the threshold on the novelty score determined from previous studies [8], $\kappa=3$, at approximately halfway through 28 April, and at midnight of 29 April. These increases in the trend of the novelty score indicate the physiological deterioration of the patient, corresponding to increases in heart rate (tachycardia) and breathing rate (tachypnoea), in conjunction with decreases in oxygen saturation. These abnormalities precede the emergency admission of the patient to the ICU halfway through 29 April.

A second case study is shown in Figure 4, in which the patient is admitted to the ICU at the beginning of their stay, during the 24 April, and who is then subsequently monitored during recovery for 4 days. It may again be seen that the automatically-acquired data from the monitor closely match the less frequent manual observations. Manual observations resume towards the end of the 25 April, with monitoring resuming halfway through 26 April.

The novelty scores for this case study show that the patient remains in an unstable condition for several days after their return from the ICU on 25 April, with particularly high novelty scores corresponding to deranged physiology (notable, tachycardia) during the 27 April. It may be seen from the figure that the trend of the novelty scores decreases as the patient recovers throughout the monitoring period.

\section{Conclusions, discussion, and future studies}

This paper has introduced preliminary results from an ongoing investigative study in the use of automatic, probabilistic monitoring for ambulatory patients. An analysis of the existing manual methods of care indicate that a significant number of events corresponding to physiological deterioration could be missed, possibly due to human error when attempting to perform manual scoring of vital signs. In comparison, the proposed method shows that abnormalities in patient condition can be detected automatically when novelty scores exceed a probabilistic threshold, and that "patient state" is represented by the level of the (smoothed) novelty score.

\subsection{Obstacles}

The use of hospital patients has enabled the technology to be developed for real-time acquisition of data from ambulatory patients, such as required for home monitoring. Most notably, the largest obstacles to overcome in this investigative phase have been (i) the reliability of the transmission of data from the patient PDA to the Central Station via the wireless network, and (ii) the compliance of patients to ensure that they wear the sensors. The former problem has been solved through improved transmission protocols in software, while the latter is an on-going "human factor" which requires careful coaching by the clinicians involved in caring for the patients. As mentioned previously, the original intention for the project was to employ ECG monitoring in parallel with the use of the pulse oximeter, such that further sources of heart rate and respiration rate could be obtained, and subsequently fused with those from the pulse oximeter. However, patient intolerance of chest-mounted electrodes was such that ECG monitoring was stopped during the investigation. This problem could be exacerbated in the case of home monitoring, in which patient discomfort at wearing sensors becomes more pronounced as the duration of monitoring increases, and as the patient becomes less acutely ill.

\subsection{Technological improvements}

While the monitoring technique described in this paper has been demonstrated to be correlated to patient status, further work is required in order to improve its tolerance to artefact and noise. In particular, a full evaluation of the false-alert rate of the technology is proposed for the next step of the investigation, when all patient data have been accumulated.

The probabilistic approach can also be improved, and work in concurrent, related studies has suggested that recent improvements in the manner in which "abnormality" is modelled, such as using extreme value statistics (a branch of statistics used to model extremal, rare events in data), could be exploited for the use of the system in home monitoring. 

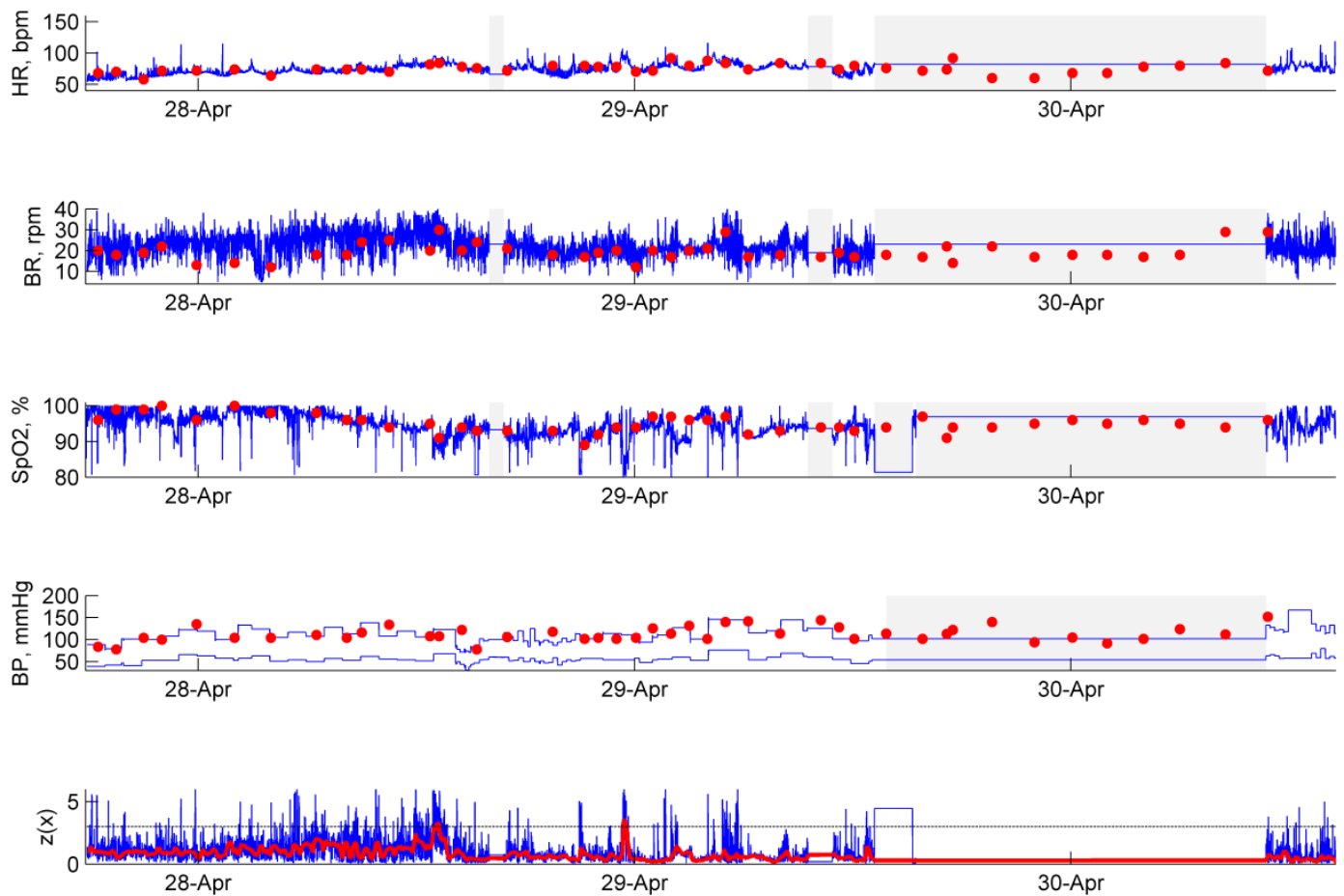

Figure 3: A deteriorating ambulatory patient, with manual observations shown in red. Unprocessed and smooth novelty scores are shown in blue and red, respectively. A novelty threshold is shown at $z(\mathrm{x})=\kappa=3$ by a horizontal black line. Unmonitored periods are shown with grey backgrounds.
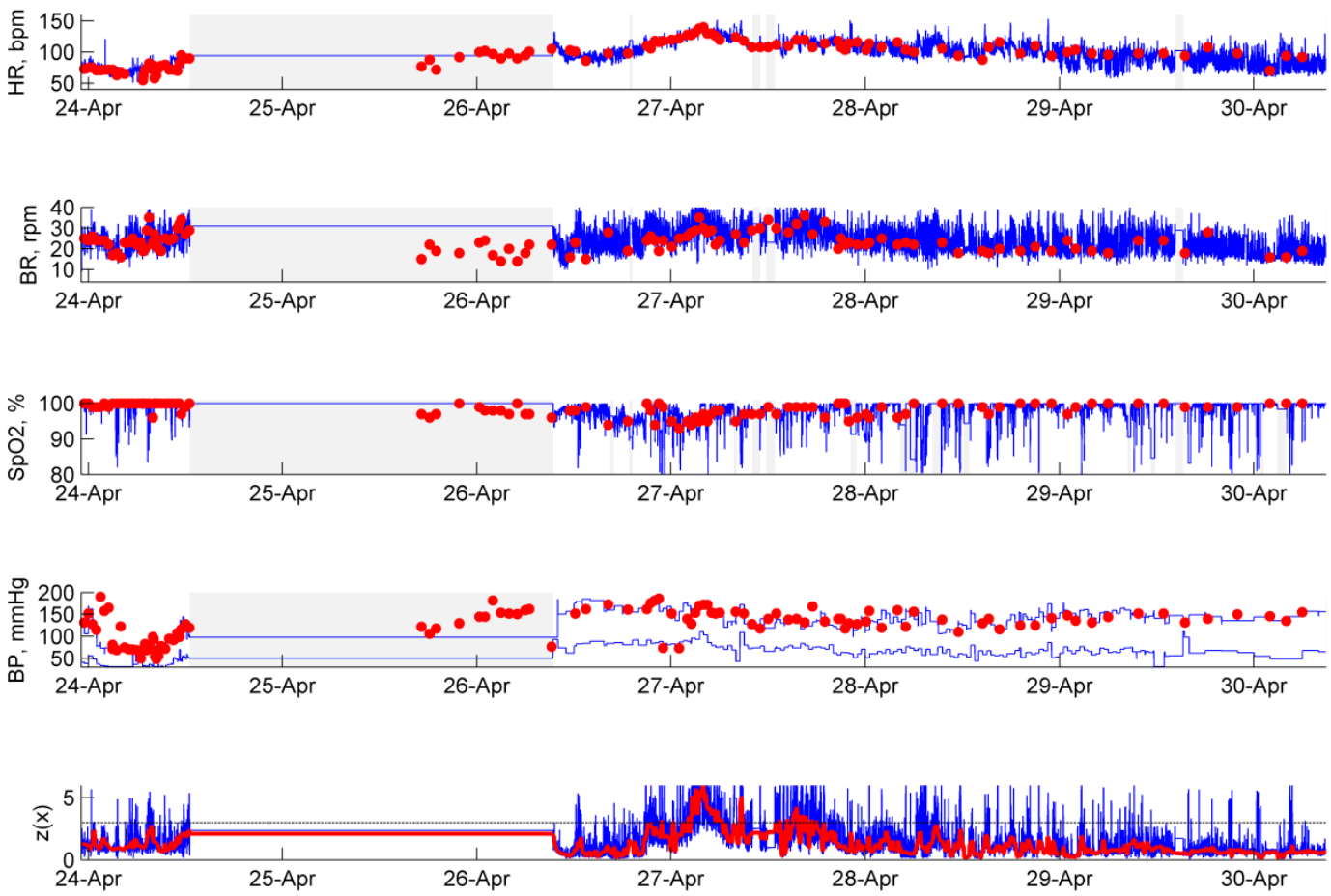

Figure 4: A deteriorated patient with post-ICU ambulatory monitoring, with colours as shown in the previous figure. 


\section{Acknowledgements}

Drs. David Clifton and David Meredith were supported by the Centre of Excellence in Personalised Healthcare funded by the Wellcome Trust and the EPSRC under grant number WT 088877/Z/09/Z. Dr. Lei Clifton was supported by the NIHR Biomedical Research Centre, Oxford. Sara Khalid was supported by the Rhodes Trust.

\section{References}

[1] C. M. Bishop, "Novelty detection and neural network validation," Proceedings of IEE Conference on Vision and Image Signal Processing, vol. 141, no. 4, pp. 217222,1994

[2] C. M. Bishop, "Pattern recognition and machine learning”, Springer-Verlag, 2006.

[3] Clifton, D.A., Hugueny, S., and Tarassenko, L., "Novelty Detection with Multivariate Extreme Value Statistics", Journal of Signal Processing Systems, 2010.

[4] M. Hravnak, L. Edwards, A. Clontz, C. Valenta, M.A. DeVita, and M.R. Pinsky, "Defining the incidence of cardiorespiratory instability in patients in stepdown units using an electronic integrated monitoring system," Archives of Internal Medicine, vol. 168, no. 12, pp. 1300-1308, 2008.

[5] Q. Li, R.G. Mark, and G.D. Clifford, "Robust heart rate estimation from multiple asynchronous noisy sources using signal quality indices and a kalman filter," IOP Physiological Measurement, vol. 29, pp. 15-32, 2008.

[6] National Patient Safety Association, "Safer care for acutely ill patients: Learning from serious accidents," Tech. Rep., NPSA, 2007.

[7] J.T. Sharpley and J.C. Holden, "Introducing an early warning scoring system in a district general hospital," Nursing in Critical Care, vol. 9, pp. 98-103, 2004.

[8] L. Tarassenko, A. Hann, and D. Young, "Integrated monitoring and analysis for early warning of patient deterioration," British Journal of Anaesthesia, vol. 98, no. 1, pp. 149-152, 2007. 